\title{
Volatile anesthetics in preventing acute kidney injury after cardiac surgery: A systematic review and meta-analysis
}

\author{
Jieru Cai, MD, ${ }^{\mathrm{a}, \mathrm{c}}$ Rende Xu, MD, PhD, ${ }^{\mathrm{b}}$ Xiaofang Yu, MD, PhD, ${ }^{\mathrm{a}, \mathrm{c}, \mathrm{d}}$ Yi Fang, MD, PhD, ${ }^{\mathrm{a}, \mathrm{c}, \mathrm{d}}$ and \\ Xiaoqiang Ding, $\mathrm{MD}, \mathrm{PhD}^{\mathrm{a}, \mathrm{c}, \mathrm{d}}$
}

\begin{abstract}
Objective: Acute kidney injury is a common clinical complication of cardiac surgery. Volatile anesthetics have been shown to protect against it in animal experiments. Clinically, however, the effect of volatile anesthetics has been unclear. We conducted a systematic review and meta-analysis of randomized, controlled trials to explore whether volatile anesthetics could provide renal protection to patients undergoing cardiac surgery.
\end{abstract}

Methods: Randomized, controlled trials were identified in PubMed, Ovid, Excerpta Medica Database, Cochrane Library, Current Controlled Trials Register, reviews, and reference lists of relevant articles. Ten trials with 1600 total participants were eligible. Data were analyzed with both fixed- and random-effects models.

\begin{abstract}
Results: Relative to control data, volatile anesthetics significantly reduced acute kidney injury incidence (relative risk [RR], 0.65; 95\% confidence interval [CI], 0.43-0.97; $P=.04$ ). Although there was no significant difference between groups in absolute postoperative serum creatinine level and mortality, patients receiving volatile anesthetics had significantly (or borderline) lower increase in serum creatinine level from baseline on the first (weighted mean difference, $-0.04 \mathrm{mg} / \mathrm{dL} ; 95 \% \mathrm{CI},-0.07$ to $-0.01 \mathrm{mg} / \mathrm{dL} ; P=.002$ ) and second (weighted mean difference, $-0.07 \mathrm{mg} / \mathrm{dL}, 95 \% \mathrm{CI},-0.14$ to $-0.00 \mathrm{mg} / \mathrm{dL} ; P=.05$ ) postoperative days and reduced incidences of prolonged intensive care unit stay (RR, 0.46; 95\% CI, 0.34-0.64; $P<.001$ ) and hospitalization (RR, 0.47; 95\% CI, 0.27-0.83; $P=.009$ ).
\end{abstract}

Conclusions: Current evidence shows that volatile anesthetics may provide renal protection in patients undergoing cardiac surgery and supports further randomized, controlled trials with larger sample sizes and high methodologic quality. (J Thorac Cardiovasc Surg 2014;148:3127-36)

See related commentary on pages $3137-8$.

Acute kidney injury (AKI), characterized by a rapid fall in glomerular filtration rate and accumulation of nitrogenous waste products, remains a common complication of heart surgery. ${ }^{1,2}$ Postoperative AKI affects as many as $45 \%$ of patients undergoing cardiac surgery and has been associated with prolonged hospitalization and increased mortality. ${ }^{3-5}$ In clinical practice, renal ischemia-reperfusion (IR) injury is a major factor responsible for postoperative

\footnotetext{
From the Departments of Nephrology ${ }^{\mathrm{a}}$ and Cardiology, ${ }^{\mathrm{b}}$ Zhongshan Hospital, Fudan University, Shanghai, China; Kidney and Dialysis Institute of Shanghai, ${ }^{\mathrm{c}}$ Shanghai, China; and Kidney and Blood Purification Laboratory of Shanghai, ${ }^{\mathrm{d}}$ Shanghai, China.

Supported by the Major Project of Basic Research of Technology Committee in Shanghai of China (Grant No. 12DJ1400200) and the National Natural Science Foundation of China (Grant No. 81300208).

Disclosures: Authors have nothing to disclose with regard to commercial support. C.J. and X.R. contributed equally to this work.

Received for publication March 24, 2014; revisions received June 6, 2014; accepted for publication July 15, 2014; available ahead of print Sept 11, 2014.

Address for reprints: Ding Xiaoqiang, MD, PhD, Department of Nephrology, Zhongshan Hospital, Fudan University, 180 Fenglin Rd, Shanghai 200032, China (E-mail: ding.xiaoqiang@zs-hospital.sh.cn). $0022-5223 / \$ 36.00$

Copyright (C) 2014 Published by Elsevier Inc. on behalf of The American Association for Thoracic Surgery

http://dx.doi.org/10.1016/j.jtcvs.2014.07.085
}

AKI. ${ }^{6}$ Agents that can attenuate IR injury have therefore been sought and studied.

Volatile anesthetics are commonly used for general anesthesia during surgery to induce and maintain hypnosis, analgesia, and amnesia and to produce mild muscle relaxation. In addition to their anesthetic effects, volatile anesthetics are believed to have significant nonanesthetic physiologic effects. ${ }^{8,9}$ An increasing body of evidence has convincingly demonstrated that volatile anesthetics can protect against IR injury in the heart. ${ }^{8,10}$ The mechanisms of cardioprotective effects have been suggested to be related to ischemic preconditioning effect induced by volatile anesthetics. ${ }^{11,12}$ Because the preconditioning holds the characteristic of cross-tolerance, the protective effects of volatile anesthetics may extend to other organ systems subject to IR injury. ${ }^{9}$ Volatile anesthetics have therefore been hypothesized to have renal protection potential in cardiac surgery. Moreover, the protection of volatile anesthetics against IR-induced renal injury has been confirmed by a number of animal studies. ${ }^{13-15}$ The clinical studies regarding the protective effects of volatile anesthetics against AKI in patients undergoing cardiac surgery have been limited, however, and the results have remained controversial. ${ }^{16,17}$ In this meta-analysis of randomized controlled trials (RCTs), we aimed to assess the effectiveness of volatile anesthetics for renal protection in patients undergoing cardiac surgery. 


$$
\begin{aligned}
& \text { Abbreviations and Acronyms } \\
& \begin{aligned}
\text { AKI } & =\text { acute kidney injury } \\
\text { CI } & =\text { confidence interval } \\
\text { ICU } & =\text { intensive care unit } \\
\text { IR } & =\text { ischemia-reperfusion } \\
\text { RCT } & =\text { randomized, controlled trial } \\
\text { RR } & =\text { relative risk } \\
\text { TIVA } & =\text { total intravenous anesthesia } \\
\text { WMD } & =\text { weighted mean difference }
\end{aligned}
\end{aligned}
$$

\section{MATERIALS AND METHODS \\ Search Strategy}

The literature search was performed on PubMed, the Ovid information system, the Excerpta Medica Database (EMBASE), and the Cochrane Library through January 2014. Complex search strategies were formulated with the following Medical Subject Headings (MeSH) terms and text words: inhalation anesthetic, halogenated anesthetic, volatile anesthetic, sevoflurane, desflurane, isoflurane, enflurane, halothane, cardiac surgery, heart surgery, mitral valve, aortic valve, coronary surgery, coronary artery bypass graft, and cardiopulmonary bypass. To find any studies missed by these literature searches, we hand searched reference lists of all eligible studies and relevant review articles. In addition, we searched for unpublished and ongoing trials in clinical trial registries (ClinicalTrials.gov, Controlledtrials.com, and the World Health Organization International Clinical Trials Registry Platform). Searches were not restricted by language, date published, or publication status. Duplicate reports were eliminated.

\section{Study Selection}

We included studies when the following criteria were met: (1) RCTs, (2) studies conducted in a cardiac surgical population, (3) intervention consisting of an anesthesia plan including administration of any volatile anesthetics without restriction in dose and time of administration, (4) patients in the comparator arm receiving total intravenous anesthesia (TIVA), (5) availability of measurements of serum creatinine concentrations at baseline and follow-up or postoperative AKI incidence. Trials were excluded if they did not conform to these criteria or when children were involved.

\section{Data Extraction}

Two reviewers (D. X. and C. J.) assessed the eligibility of studies in duplicate and independently by means of a standardized form developed for this purpose. Disagreements were adjudicated by a third reviewer. Data extraction was done by the same observers with a standardized data extraction form developed for this study. The following information was extracted from each study: study and patient characteristics, anesthetic protocol, volatile anesthetic dosage and treatment duration, choice and dosage of intravenous anesthetics, duration of cardiopulmonary bypass, cardioplegic technique, hypothermic condition, target mean arterial pressure, inotrope therapy, the incidence of AKI, mortality, rates of prolonged intensive care unit (ICU) stay and hospitalization, and levels of kidney injury biomarkers (serum creatinine and cystatin C). Several studies met our inclusion criteria but were missing data vital to our analyses (eg, means and SDs); we therefore contacted the authors to obtain raw data whenever possible. For studies involving 2 intervention arms that had 2 different volatile or intravenous anesthetics, data were analyzed according methods described in the Cochrane Handbook for Systematic Reviews of Interventions.

\section{Quality Assessment}

According to standard criteria established by Jüni and colleagues, ${ }^{18} 2$ independent reviewers (D. X. and C. J.) assessed aspects of the reported methodologic quality of each included study pertaining to generation of randomized sequences, concealment of treatment allocation schedules, blinding of outcome assessments, and adequacy of follow-up.

\section{Statistical Analysis}

Statistical analyses in this study were carried out with RevMan software (version 5.2; The Cochrane Collaboration; Oxford, UK). The primary outcomes of this study were the level and change of creatinine after surgery and the development of AKI. For assessment of the change in the creatinine level from baseline, the unbiased estimator of variance method in the Cochrane Handbook was used to calculate the SD. In studies in which medians were reported, means and SDs were estimated if we could not get the raw data from the authors. ${ }^{19}$ Continuous outcomes were calculated as weighted mean differences (WMDs), and dichotomous outcomes were calculated as relative risks (RRs), with their respective $95 \%$ confidence intervals (CIs). The pooled WMD and common RR with 95\% CI values for all studies were computed according to both fixed- and randomeffects models; the latter was more conservative where heterogeneity beyond that expected by chance alone was encountered. The $\chi^{2}$ test was used to estimate heterogeneity across the studies, with a value of $50 \%$ or more indicating a substantial level of inconsistency. A planned subgroup analysis was performed according to the choice of volatile anesthetic.

\section{RESULTS}

\section{Search Strategy}

The initial literature search identified 2322 potentially relevant citations (Figure 1), of which 2218 were excluded after independent review of the title and abstract. The remaining 104 articles were considered of interest and examined in full text. Of these, 68 were excluded from the meta-analysis (review articles, non-RCTs, irrelevant to our aim). After assessment in more detail, 26 of the remaining 36 RCTs were excluded because 25 did not report postoperative serum creatinine value or incidence of AKI and 1 lacked a TIVA control group. The remaining 10 RCTs were included in the systematic review. ${ }^{16,20-28}$

\section{Characteristics of Included Studies}

Table 1 summarizes the main features of the included studies. A total of 1600 patients ( 860 allocated to receive volatile anesthetics and 740 receiving TIVA in their anesthesia plan) were enrolled in the 10 studies, and the sample sizes were 22 to 354 participants of each study. Six trials were performed on patients undergoing coronary surgery, and the other 4 studies were performed on patients undergoing aortic or mitral valve surgery. Volatile anesthetics were administrated throughout the procedure in 7 of the involved studies, whereas in 3 of them volatile anesthetics administered for a short time and only before, during, or after the cardiopulmonary bypass. The minimum alveolar concentration of volatile anesthetics was always at least 0.15 and ranged from 0.15 to 2. There were no significant differences between the groups with regard to age, sex, comorbidities (hypertension and diabetes), and preoperative renal status.

The intraoperative and postoperative characteristics of the involved studies are presented in Table 2. Most studies were performed on patients undergoing cardiac surgery 


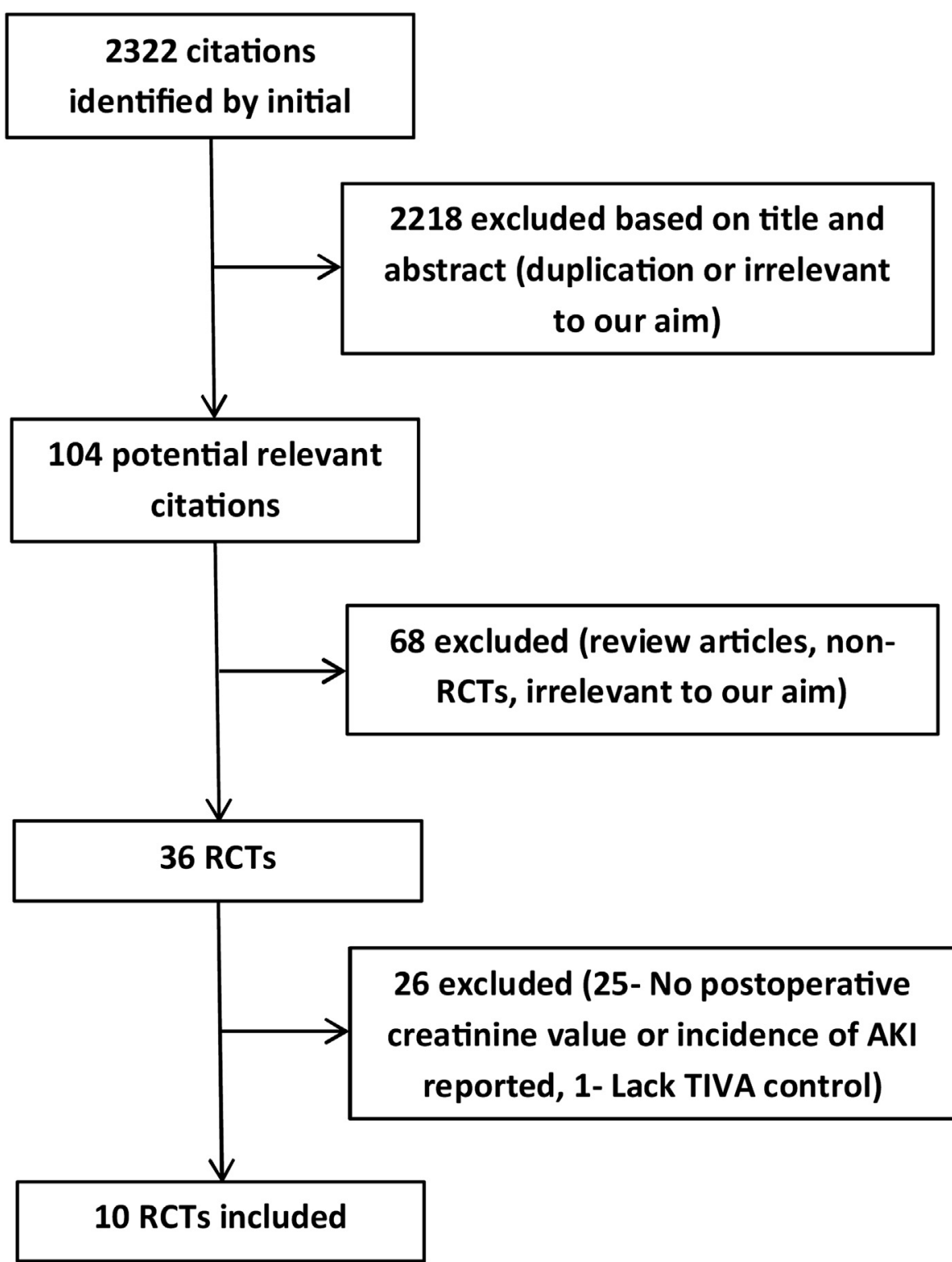

FIGURE 1. Flow diagram of trial search and selection progress. RCT, Randomized, controlled trial; AKI, acute kidney injury; TIVA, total intravenous anesthesia.

with cardiopulmonary bypass, and only 1 study investigated patients undergoing off-pump coronary artery bypass grafting. ${ }^{23}$ In these studies, cardiopulmonary bypass was conducted under hypothermic conditions $\left(28^{\circ} \mathrm{C}-36^{\circ} \mathrm{C}\right)$, and the cardiopulmonary bypass time was comparable between the volatile anesthetic and control groups $(P=.21)$. Four studies mentioned the cardioplegic techniques; in all of these, cold blood or cardioplegic solution was applied. In most trials, the target mean arterial pressure was set to greater than 50 to $65 \mathrm{~mm} \mathrm{Hg}$; otherwise, inotropic or vasoactive support would be required. Compared with TIVA group, use of volatile anesthetics was also associated with a significant reduction in need for inotrope therapy
$(P<.001)$. Postoperatively, 7 trials reported the incidence of AKI according to the level of serum creatinine or cystatin C.

\section{Quality Assessment}

Table 3 describes the methodologic quality of the RCTs. The overall methodologic quality of the included RCTs was good. All included studies randomly allocated their patients, and 8 of them described adequate methods to generate randomized sequences and had concealed the randomization allocations. Only 2 trials did not confirm the use of blinded outcome assessors. All 10 trials reported adequate details of withdrawals and dropouts in each group. 
TABLE 1. Main features of studies included in meta-analysis (volatile anesthetic group and total intravenous anesthesia group)

\begin{tabular}{|c|c|c|c|c|c|c|c|c|c|c|c|c|c|}
\hline \multirow[b]{2}{*}{ Study } & \multirow[b]{2}{*}{$\begin{array}{l}\text { Sample } \\
\text { size }(n)\end{array}$} & \multirow[b]{2}{*}{$\begin{array}{l}\text { Mean } \\
\text { age }(y)\end{array}$} & \multirow[b]{2}{*}{$\begin{array}{c}\text { Male } \\
(\%)\end{array}$} & \multicolumn{2}{|c|}{ Comorbidities } & \multirow[b]{2}{*}{$\begin{array}{c}\text { Surgical } \\
\text { procedure }\end{array}$} & \multirow[b]{2}{*}{$\begin{array}{c}\text { Volatile } \\
\text { anesthetic }\end{array}$} & \multirow[b]{2}{*}{$\begin{array}{l}\text { Adminis- } \\
\text { tration }\end{array}$} & \multirow[b]{2}{*}{ MAC } & \multirow[b]{2}{*}{ TIVA } & \multirow[b]{2}{*}{ Dosage } & \multicolumn{2}{|c|}{$\begin{array}{l}\text { Baseline creatinine } \\
(\mathrm{mg} / \mathrm{dL} ; \text { mean } \pm \mathrm{SD})\end{array}$} \\
\hline & & & & $\begin{array}{r}\text { HTN } \\
\text { (no.) }\end{array}$ & $\begin{array}{c}\text { Diabetes } \\
\text { (no.) }\end{array}$ & & & & & & & $\begin{array}{c}\text { Volatile } \\
\text { anesthetic }\end{array}$ & TIVA \\
\hline $\begin{array}{l}\text { Bignami et al, } \\
2012\end{array}$ & $50 / 50$ & $67 / 66$ & $74 / 78$ & $28 / 29$ & $5 / 1$ & MVR & Sevo & $\begin{array}{l}\text { Pre-CPB, } \\
\text { post-CPB }\end{array}$ & $0.5-2$ & Propofol & $2-3 \mathrm{mg} / \mathrm{kg} / \mathrm{h}$ & $1.05 \pm 0.69$ & $0.98 \pm 0.25$ \\
\hline Cho et al, 2009 & $15 / 15$ & $57 / 48$ & $20 / 20$ & $7 / 6$ & $2 / 3$ & $\begin{array}{l}\text { MVR, } \\
\text { AVR }\end{array}$ & Sevo & $\begin{array}{l}\text { Whole } \\
\text { procedure }\end{array}$ & $0.75-1.25$ & $\begin{array}{r}\text { Sufentanil }+ \\
\text { midazolam }\end{array}$ & $\begin{array}{l}2.0-2.2 \\
\mu \mathrm{g} / \mathrm{kg} / \mathrm{h} ; \\
0.08 \\
\mathrm{mg} / \mathrm{kg} / \mathrm{h}\end{array}$ & NR & NR \\
\hline $\begin{array}{l}\text { De Hert et al, }{ }^{22} \\
\quad 2004\end{array}$ & $160 / 160$ & $67 / 67$ & $82 / 81$ & NR & $44 / 46$ & $\begin{array}{r}\text { Coronary } \\
\text { surgery }\end{array}$ & Sevo; Des & $\begin{array}{l}\text { Whole } \\
\text { procedure }\end{array}$ & $0.15-1$ & $\begin{array}{l}\text { Propofol, } \\
\text { midazolam }\end{array}$ & $\begin{array}{c}2-4 \mu \mathrm{g} / \mathrm{mL} \\
0.1-1.5 \\
\mu \mathrm{g} / \mathrm{kg} / \mathrm{mL}\end{array}$ & NR & NR \\
\hline $\begin{array}{l}\text { Guarracino } \\
\text { et al, },^{23} 2006\end{array}$ & $57 / 55$ & $69 / 69$ & $86 / 78$ & NR & $14 / 13$ & OPCAB & Des & $\begin{array}{l}\text { whole } \\
\text { procedure }\end{array}$ & $0.5-2$ & Propofol & $2-3 \mathrm{mg} / \mathrm{kg} / \mathrm{h}$ & $1.3 \pm 0.36$ & $1.3 \pm 0.80$ \\
\hline $\begin{array}{l}\text { Jovic et al, }{ }^{24} \\
\quad 2012\end{array}$ & $11 / 11$ & $63 / 63$ & $45 / 73$ & $8 / 7$ & $1 / 2$ & AVR & Sevo & $\begin{array}{l}\text { Whole } \\
\text { procedure }\end{array}$ & $1-2$ & Propofol & $6-10 \mathrm{mg} / \mathrm{kg} / \mathrm{h}$ & NR & NR \\
\hline $\begin{array}{l}\text { Julier et al, }^{16} \\
2003\end{array}$ & $37 / 35$ & $62 / 65$ & $84 / 80$ & $26 / 22$ & $9 / 9$ & CABG & Sevo & $\begin{array}{c}\text { During CPB } \\
10 \mathrm{~min}\end{array}$ & 2 & Propofol & $2-5 \mu \mathrm{g} / \mathrm{mL}$ & $0.98 \pm 0.24$ & $0.97 \pm 0.23$ \\
\hline $\begin{array}{l}\text { Landoni et al, }{ }^{25} \\
\quad 2007\end{array}$ & $59 / 61$ & $62 / 59$ & $59 / 50$ & $17 / 19$ & NR & $\begin{array}{l}\text { MV } \\
\quad \text { surgery }\end{array}$ & Des & $\begin{array}{c}\text { During CPB } \\
30 \mathrm{~min}\end{array}$ & $0.5-2$ & Propofol & $2-3 \mathrm{mg} / \mathrm{kg} / \mathrm{h}$ & $0.9 \pm 0.24$ & $0.9 \pm 0.25$ \\
\hline $\begin{array}{l}\text { Lorsomradee } \\
\text { et al, }{ }^{26} 2006\end{array}$ & $160 / 160$ & $67 / 66$ & $86 / 75$ & NR & $39 / 50$ & CABG & Sevo & $\begin{array}{l}\text { Whole } \\
\text { procedure }\end{array}$ & $0.25-1$ & Propofol & $2-4 \mu \mathrm{g} / \mathrm{mL}$ & $1.1 \pm 0.3$ & $1.0 \pm 0.2$ \\
\hline $\begin{array}{l}\text { Story et al, }{ }^{27} \\
\quad 2001\end{array}$ & $236 / 118$ & $67 / 65$ & $81 / 84$ & NR & $57 / 42$ & CABG & Sevo; Iso & $\begin{array}{l}\text { Whole } \\
\text { procedure }\end{array}$ & $\begin{array}{l}\text { Sevo, 0.5-2; } \\
\text { Iso, } 0.25-1\end{array}$ & Propofol & $1-8 \mu \mathrm{g} / \mathrm{mL}$ & $\begin{array}{r}\text { Sevo, } 1.13 \pm 0.28 \\
\text { Iso, } 1.09 \pm 0.22\end{array}$ & $1.10 \pm 0.22$ \\
\hline $\begin{array}{l}\text { Tritapepe et al, } \\
2007\end{array}$ & $75 / 75$ & $64 / 66$ & $84 / 80$ & NR & $18 / 13$ & CABG & Des & $\begin{array}{l}\text { Whole } \\
\text { procedure }\end{array}$ & 1 & Propofol & $2-3 \mu \mathrm{g} / \mathrm{mL}$ & $1.1 \pm 0.42$ & $1.1 \pm 0.30$ \\
\hline
\end{tabular}

$\overline{H T N}$, Hypertension; $M A C$, minimum alveolar concentration; TIVA, total intravenous anesthesia; $M V R$, mitral valve replacement; Sevo, sevoflurane; $C P B$, cardiopulmonary bypass; $A V R$, aortic valve replacement; $N R$, not reported; $D e s$, desflurane; $O P C A B$, off-pump coronary artery bypass grafting; $C A B G$, coronary artery bypass grafting; $M V$, mitral valve; Iso, isoflurane; $S D$, standard deviation.

\section{Effects of Volatile Anesthetics on Postoperative Renal Function and Clinical Outcomes}

Incidences of AKI and renal replacement therapy. There were 7 trials included in the meta-analysis that reported the incidence of AKI. AKI was defined differently among the involved studies. Three studies used a postoperative increase in serum creatinine greater than $100 \%, 1$ study used a prospectively defied cutoff concentration of greater than $1.5 \mathrm{mg} / \mathrm{L}$ for cystatin $\mathrm{C}$, and the remaining studies regarded peak serum creatinine level greater than $1.5 \mathrm{mg} / \mathrm{dL}$ or at least $2.0 \mathrm{mg} / \mathrm{dL}$ as their primary definition of AKI. The overall results based on a fixed-effects model showed that, relative to the control group, the use of volatile anesthetics significantly reduced the risk of AKI (RR, $0.65 ; 95 \%$ CI, 0.43-0.97; $P=.04$; $I^{2}=0 \%$; Figure $2, A$ ). To investigate whether the type of volatile anesthetic would influence the effectiveness, subgroup analysis by desflurane versus sevoflurane was performed. The reduction of AKI incidence was significant with sevoflurane (RR, 0.49; 95\% CI, 0.25-0.97; $P=.04$; $I^{2}=36 \%$; Figure $2, A$ ), which was the same as the overall result. Although a reduced risk of AKI was not found in studies for the use of desflurane (RR, $0.77 ; 95 \% \mathrm{CI}$, $0.47-1.27 ; P=.30 ; I^{2}=0 \%$; Figure $2, A$ ), there was no significant difference between patients receiving these 2 types of volatile anesthetic $(P=.29)$. Furthermore, information on renal replacement therapy was available for 2 studies. No significant difference was detected in the incidence of renal replacement therapy between the volatile anesthetic and TIVA groups (RR, 1.20; 95\% CI, 0.28-5.15; $P=.81 ; I^{2}=0 \%$; Figure $\left.2, B\right)$.

Biomarkers of kidney injury. The mean levels of serum creatinine ascertained at ICU arrival and on the first and second postoperative day were assessed. The serum creatinine levels at ICU arrival (WMD, $0.00 \mathrm{mg} / \mathrm{dL} ; 95 \%$ CI, -0.04 to $0.04 \mathrm{mg} / \mathrm{dL} ; P=.94 ; I^{2}=0 \%$; Figure $3, A$ ), on the first postoperative day (WMD, $-0.01 \mathrm{mg} / \mathrm{dL}, 95 \%$ CI, -0.05 to $0.03 \mathrm{mg} / \mathrm{dl} ; P=.64 ; I^{2}=0 \%$; Figure $3, B$ ), and on the second postoperative day (WMD, -0.05 $\mathrm{mg} / \mathrm{dL} ; 95 \%$ CI, -0.10 to $0.00 \mathrm{mg} / \mathrm{dL} ; P=.07$; $I^{2}=0 \%$; Figure $3, C$ ) did not differ evidently between the volatile anesthetic and control groups. In addition, we compared mean changes from baseline in serum creatinine levels between the control and treatment groups. Relative to TIVA, the overall pooled results showed that volatile anesthetics did not bring a significant change in creatinine level at the ICU arrival (WMD, $-0.04 \mathrm{mg} / \mathrm{dL} ; 95 \% \mathrm{CI}$, -0.10 to $0.02 \mathrm{mg} / \mathrm{dL} ; P=.18 ; I^{2}=73 \%$; Figure $4, A$ ). Patients who received volatile anesthetics did, however, have significantly (or borderline) lower increase in serum creatinine level from baseline on the first postoperative day (WMD, $-0.04 \mathrm{mg} / \mathrm{dL} ; 95 \% \mathrm{CI},-0.07$ to -0.01 
TABLE 2. Intraoperative and postoperative characteristics of studies included in meta-analysis (volatile anesthetic group and total intravenous anesthesia group)

\begin{tabular}{|c|c|c|c|c|c|c|c|c|c|}
\hline \multirow[b]{2}{*}{ Study } & \multicolumn{2}{|c|}{$\begin{array}{c}\text { CPB time } \\
(\mathrm{min}, \text { mean } \pm \mathrm{SD})\end{array}$} & \multirow[b]{2}{*}{$\begin{array}{c}\text { Cardioplegic } \\
\text { technique }\end{array}$} & \multirow{2}{*}{$\begin{array}{c}\text { Hypothermic } \\
\text { condition } \\
\left({ }^{\circ} \mathrm{C}\right)\end{array}$} & \multirow[b]{2}{*}{$\begin{array}{c}\text { Target MAP } \\
\text { (mm Hg) }\end{array}$} & \multirow[b]{2}{*}{$\begin{array}{c}\text { Intraoperative } \\
\text { inotropes }\end{array}$} & \multirow[b]{2}{*}{$\begin{array}{c}\text { Postoperative } \\
\text { inotropes }\end{array}$} & \multirow[b]{2}{*}{$\begin{array}{c}\text { AKI } \\
\text { definition }\end{array}$} & \multirow[b]{2}{*}{$\begin{array}{c}\text { AKI } \\
\text { incidence }\end{array}$} \\
\hline & $\begin{array}{c}\text { Volatile } \\
\text { anesthetic }\end{array}$ & TIVA & & & & & & & \\
\hline $\begin{array}{l}\text { Bignami et al, }{ }^{20} \\
2012\end{array}$ & $104 \pm 21$ & $97 \pm 27$ & Cold blood & $32-34$ & $>65$ & $25 / 29$ & $33 / 38$ & NR & NR \\
\hline Cho et al, ${ }^{21} 2009$ & $129 \pm 24$ & $131 \pm 25$ & NR & 28 & $\begin{array}{l} \pm 20 \% \text { of } \\
\text { baseline }\end{array}$ & $5 / 6$ & $6 / 7$ & $\begin{array}{c}\mathrm{SCr}>2.0 \\
\mathrm{mg} / \mathrm{dL}\end{array}$ & $2 / 2$ \\
\hline $\begin{array}{l}\text { De Hert et al, }{ }^{22} \\
2004\end{array}$ & $97 \pm 28$ & $96 \pm 31$ & NR & 32 & $>60$ & $40 / 80$ & $49 / 91$ & $\begin{array}{c}\mathrm{SCr}>1.5 \\
\mathrm{mg} / \mathrm{dL}\end{array}$ & $15 / 17$ \\
\hline $\begin{array}{l}\text { Guarracino } \\
\text { et al, }{ }^{23} 2006\end{array}$ & NR & NR & NR & NR & NR & NR & $20 / 31$ & $\begin{array}{r}100 \% \mathrm{SCr} \\
\text { increase }\end{array}$ & $3 / 4$ \\
\hline $\begin{array}{l}\text { Jovic et al, }{ }^{24} \\
2012\end{array}$ & $90 \pm 25$ & $92 \pm 21$ & NR & 32 & $50-70$ & NR & $2 / 3$ & $\begin{array}{c}\mathrm{SCr}>1.5 \\
\mathrm{mg} / \mathrm{dL}\end{array}$ & $2 / 0$ \\
\hline $\begin{array}{l}\text { Julier et al, }{ }^{16} \\
2003\end{array}$ & $116 \pm 32$ & $106 \pm 31$ & $\begin{array}{l}\text { Cold } \\
\text { cardioplegic } \\
\text { solution }\end{array}$ & 32 & $>50$ & NR & NR & $\begin{array}{l}\text { Cystatin C } \\
>1.5 \mathrm{mg} / \mathrm{L}\end{array}$ & $2 / 10$ \\
\hline $\begin{array}{l}\text { Landoni et al, } \\
\quad 2007\end{array}$ & $83 \pm 24$ & $84 \pm 25$ & Cold blood & $32-34$ & $>65$ & $38 / 37$ & $25 / 33$ & $\begin{array}{r}100 \% \mathrm{SCr} \\
\text { increase }\end{array}$ & $10 / 13$ \\
\hline $\begin{array}{l}\text { Lorsomradee } \\
\text { et al, }{ }^{26} 2006\end{array}$ & $97 \pm 28$ & $98 \pm 29$ & NR & 36 & $60-80$ & $36 / 78$ & $44 / 90$ & NR & NR \\
\hline $\begin{array}{l}\text { Story et al, }{ }^{27} \\
2001\end{array}$ & $\begin{array}{l}\text { Sevo, } \\
\quad 92 \pm 23 \\
\text { Iso, } 98 \pm 23\end{array}$ & $101 \pm 24$ & NR & NR & $60-80$ & NR & NR & NR & NR \\
\hline $\begin{array}{l}\text { Tritapepe et al, }{ }^{28} \\
2007\end{array}$ & $90 \pm 28$ & $97 \pm 34$ & Cold blood & $32-34$ & NR & NR & $24 / 31$ & $\begin{array}{c}100 \% \mathrm{SCr} \\
\text { increase }\end{array}$ & $5 / 4$ \\
\hline
\end{tabular}

$\overline{C P B}$, Cardiopulmonary bypass; TIVA, total intravenous anesthesia; $M A P$, mean arterial pressure, $A K I$, acute kidney injury; $N R$, not reported; $S C r$, serum creatinine; Sevo, sevoflurane; Iso, isoflurane; $S D$, standard deviation.

$\mathrm{mg} / \mathrm{dl} ; P=.002 ; I^{2}=25 \%$; Figure $\left.4, B\right)$ and the second postoperative day (WMD, $-0.07 \mathrm{mg} / \mathrm{dL} ; 95 \% \mathrm{CI},-0.14$ to $0.00 \mathrm{mg} / \mathrm{dl} ; P=.05 ; I^{2}=72 \%$; Figure $4, C$ ). Moreover, there were 2 studies that analyzed cystatin $\mathrm{C}$, a more sensitive marker of renal injury, in both groups postoperatively. Results showed that the lower level of cystatin C on the first postoperative day associated with volatile anesthetics was of borderline statistical significance (WMD, $-0.10 \mathrm{mg} / \mathrm{L}$; $95 \% \mathrm{CI},-0.21$ to $0.00 \mathrm{mg} / \mathrm{dL} ; P=.06 ; I^{2}=0 \%$; Figure 3, D).
Prolonged ICU stay, prolonged hospitalization, and mortality. Information about the incidences of prolonged ICU stay ( $>48-72$ hours) and prolonged hospitalization ( $\geq 7-15$ days) were available for 4 and 3 trials involved in the meta-analysis, respectively, whereas mortalities were reported in 8 studies. Relative to TIVA, the numbers of patients who needed prolonged ICU stays (RR, 0.46; $95 \%$ CI, 0.34-0.64; $P<.001 ; I^{2}=0 \%$; Figure $\left.5, A\right)$ and prolonged hospitalization (RR, $0.47 ; 95 \% \mathrm{CI}, 0.27-0.83$; $P=.009 ; I^{2}=35 \%$; Figure $\left.5, B\right)$ were significantly lower

TABLE 3. Methodologic quality assessment of randomized controlled trials included in this study

\begin{tabular}{|c|c|c|c|c|c|}
\hline Study & $\begin{array}{c}\text { Adequate method to } \\
\text { generate randomized } \\
\text { sequence }\end{array}$ & $\begin{array}{c}\text { Adequate method } \\
\text { of allocation } \\
\text { concealment }\end{array}$ & $\begin{array}{l}\text { Blinding of } \\
\text { outcome } \\
\text { assessors }\end{array}$ & $\begin{array}{c}\text { Explanation for } \\
\text { withdrawals or } \\
\text { dropouts } \\
\end{array}$ & $\begin{array}{c}\text { All patients } \\
\text { treated in } \\
\text { assigned group }\end{array}$ \\
\hline Bignami et al, ${ }^{20} 2012$ & $\mathrm{Y}$ & $Y$ & $\mathrm{Y}$ & $\mathrm{Y}$ & Y \\
\hline Cho et al, ${ }^{21} 2009$ & $\mathrm{~N}$ & $\mathrm{~N}$ & $\mathrm{~N}$ & $\mathrm{Y}$ & $\mathrm{Y}$ \\
\hline De Hert et al, ${ }^{22} 2004$ & $\mathrm{Y}$ & $\mathrm{Y}$ & $\mathrm{Y}$ & $\mathrm{Y}$ & $\mathrm{Y}$ \\
\hline Guarracino et al, ${ }^{23} 2006$ & $\mathrm{Y}$ & $\mathrm{Y}$ & $\mathrm{Y}$ & $\mathrm{Y}$ & $\mathrm{Y}$ \\
\hline Jovic et al, ${ }^{24} 2012$ & $\mathrm{~N}$ & $\mathrm{~N}$ & $\mathrm{~N}$ & $\mathrm{Y}$ & $\mathrm{Y}$ \\
\hline Julier et al, ${ }^{16} 2003$ & $\mathrm{Y}$ & $\mathrm{Y}$ & $\mathrm{Y}$ & Y & $\mathrm{Y}$ \\
\hline Landoni et al, ${ }^{25} 2007$ & $\mathrm{Y}$ & $\mathrm{Y}$ & $\mathrm{Y}$ & $\mathrm{Y}$ & $\mathrm{Y}$ \\
\hline Lorsomradee et al, ${ }^{26} 2006$ & $\mathrm{Y}$ & $\mathrm{Y}$ & $\mathrm{Y}$ & $\mathrm{Y}$ & $\mathrm{Y}$ \\
\hline Story et al, ${ }^{27} 2001$ & $\mathrm{Y}$ & $\mathrm{Y}$ & $\mathrm{Y}$ & $\mathrm{Y}$ & $\mathrm{Y}$ \\
\hline Tritapepe et al, ${ }^{28} 2007$ & Y & $\mathrm{Y}$ & $\mathrm{Y}$ & $\mathrm{Y}$ & $\mathrm{Y}$ \\
\hline
\end{tabular}




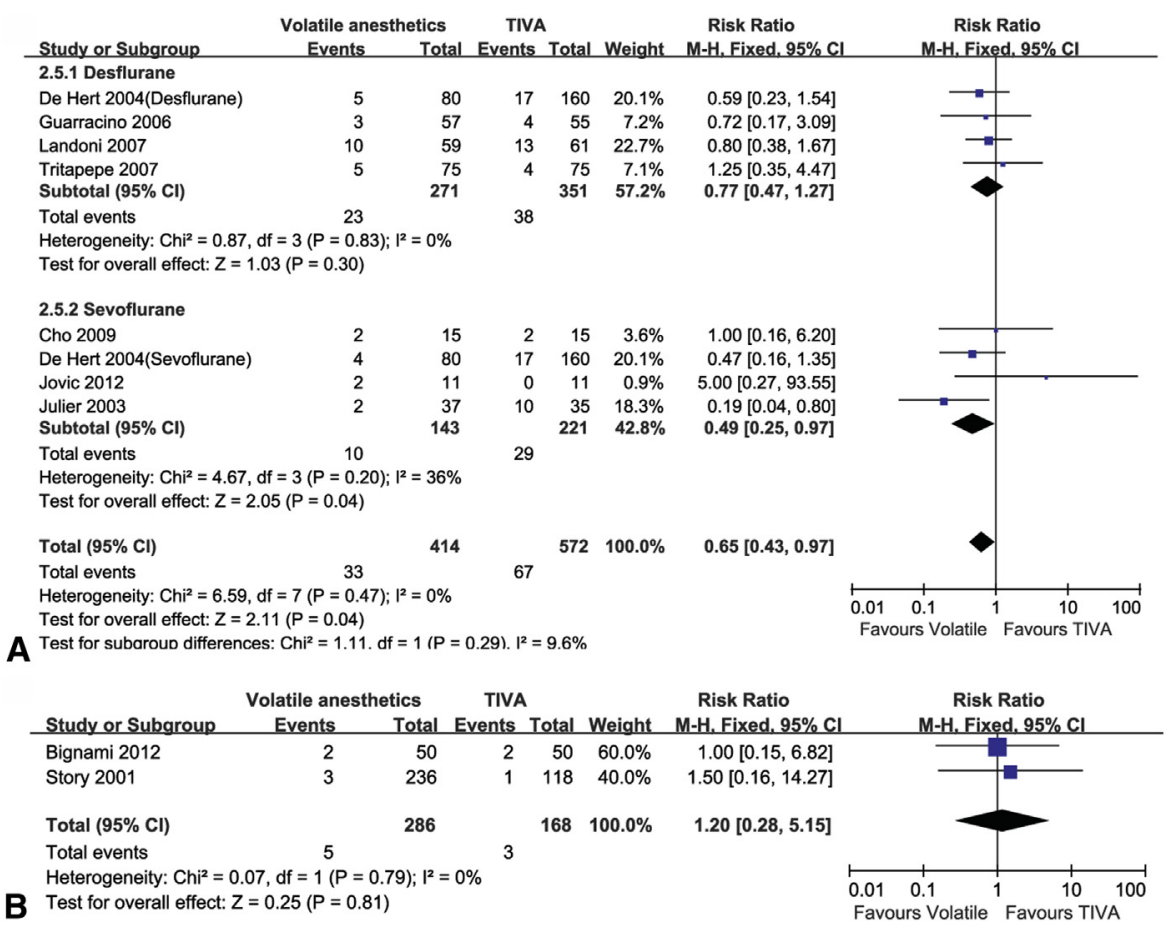

FIGURE 2. Forest plot of relative risks in the incidence of AKI (A) and renal replacement therapy (B). TIVA, Total intravenous anesthesia; $M-H$, Mantel-Haenszel (log-rank); $C I$, confidence interval.

with the volatile anesthetics. There was, however, no statistically significant difference in the all-cause mortality between the groups (RR, $0.44 ; 95 \% \mathrm{CI}, 0.14-1.33 ; P=.14$; $I^{2}=0 \%$; Figure $5, C$ ).

\section{DISCUSSION}

In this meta-analysis of $10 \mathrm{RCTs}$ involving 1600 patients who underwent heart surgery, our results generally indicated a positive effect of volatile anesthetic use on renal function and relevant clinical outcomes. Our primary outcomes were the differences between volatile anesthetics and TIVA groups in the incidence of AKI and the level and change of renal injury biomarkers during follow-up. We found that volatile anesthetics significantly improved the change from baseline in the level of serum creatinine and were associated with obvious reductions in the incidence of AKI, although there were no differences in the absolute serum creatinine levels and the rate of renal replacement therapy between the control and treatment groups. Moreover, the pooled results of these trials also indicated a benefit of volatile anesthetics for significantly decreasing the proportion of patients with prolonged ICU stay and hospitalization and demonstrated a slight trend toward reduced all-cause mortality.

In addition to their wide use in clinical practice for general anesthesia, volatile anesthetics have been found to induce a beneficial effect termed anesthetic preconditioning, which shares several common mechanisms of action with ischemic preconditioning. ${ }^{11,12,29}$ Because of their protective effects on organs under IR injury through anesthetic preconditioning, volatile anesthetics have primarily been reported to have cardioprotective properties and to ameliorate heart IR injury in cardiac and vascular interventions. AKI is another frequent complication of heart surgery and is also mainly caused by IR damage. Because ischemic preconditioning has been demonstrated in organs other than the heart, anesthetic preconditioning might also offer the potential for renal protection in cardiac surgery. Furthermore, in addition to anesthetic preconditioning, volatile anesthetics might protect the kidney from IR injury by providing more stable hemodynamic responses. ${ }^{8,30}$ It has been confirmed that, in addition to cardiopulmonary bypassinduced ischemia, hemodynamic instability also plays important role in the pathogenesis of postoperative AKI. ${ }^{31}$ In our analysis of the characteristics of the involved studies, significant reduction in need for intraoperative and postoperative inotrope therapy was found in the volatile anesthetic group, which confirmed that volatile anesthetic administration was associated with better hemodynamic stability.

Our results demonstrated that volatile anesthetics administration significantly reduced the incidence of AKI after cardiac surgery. There was no uniformly accepted definition of postoperative AKI (Table 2). A relative increase greater than $100 \%$ from baseline in serum creatinine or an absolute level greater than 1.5 to $2.0 \mathrm{mg} / \mathrm{dL}$ after cardiac surgery was used in most of the included trials. Only in the 


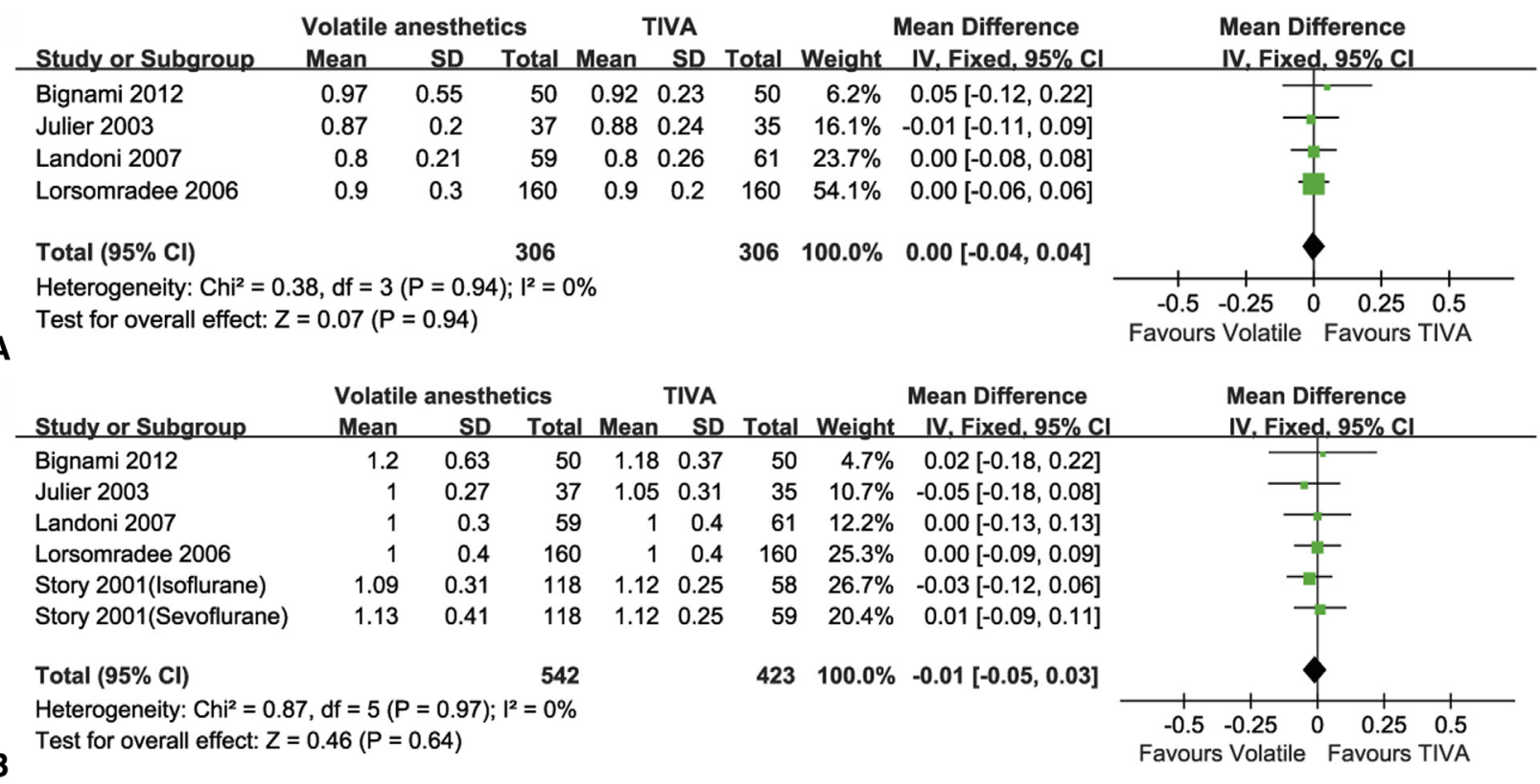

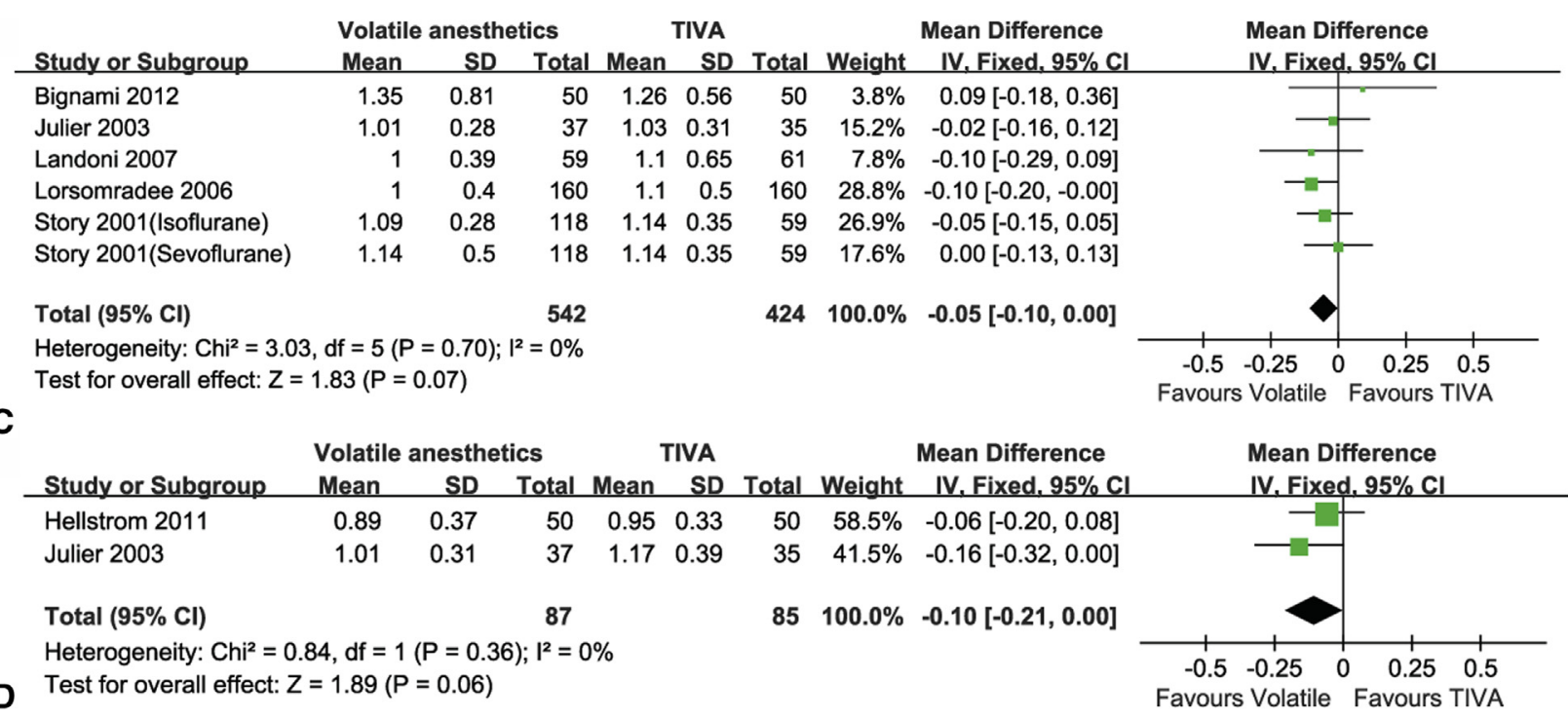

FIGURE 3. Forest plot of weighted mean differences in the levels of renal biomarkers. A, Serum creatinine level at intensive care unit arrival. $\mathrm{B}$, Serum creatinine level on the first postoperative day. C, Serum creatinine level on the second postoperative day. D, Serum cystatin C level on the first postoperative day. TIVA, Total intravenous anesthesia; $I V$, instrumental variable; $C I$, confidence interval; $S D$, standard deviation.

study by Julier and colleagues ${ }^{16}$ was postoperative renal dysfunction defined according to the level of cystatin C. Although no significant statistical heterogeneity was found in the meta-analysis of AKI incidence, there would be no significant difference in the rate of AKI between patients with volatile anesthetics and those with TIVA when we excluded the data from the study of Julier and colleagues. ${ }^{16}$ To address the variability related to the absence of a uniform definition of AKI, we compared the incidence of renal replacement therapy between the treatment and control groups. Supportive renal replacement therapy, the only treatment option currently available for AKI, has also been recommended as a hard end point in studies of AKI after cardiac surgery. ${ }^{32}$ Only 2 studies reported the rate of renal replacement therapy, however, and no significant improvement in the rate of renal replacement therapy was found.

We evaluated postoperative renal function with serum creatinine value (both absolute level and change from baseline). When analyzed in term of the absolute serum creatinine level, there was no significant difference between the treatment and control groups. It was noted, however, that patients receiving volatile anesthetics had an apparent trend toward lower serum creatinine level on the second 


\begin{tabular}{|c|c|c|c|c|c|c|c|c|c|}
\hline \multirow[b]{2}{*}{ Study or Subgroup } & \multicolumn{3}{|c|}{ Volatile anesthetics } & \multicolumn{3}{|c|}{ TIVA } & & \multirow{2}{*}{$\begin{array}{l}\text { Mean Difference } \\
\text { IV. Random, } 95 \% \mathrm{Cl}\end{array}$} & \multirow{2}{*}{$\begin{array}{c}\text { Mean Difference } \\
\text { IV. Random, } 95 \% \mathrm{CI}\end{array}$} \\
\hline & Mean & SD & Total & Mean & SD & Total & Weight & & \\
\hline Bignami 2012 & -0.08 & 0.41 & 50 & -0.06 & 0.15 & 50 & $14.6 \%$ & $-0.02[-0.14,0.10]$ & \\
\hline Julier 2003 & -0.11 & 0.14 & 37 & -0.09 & 0.15 & 35 & $25.0 \%$ & $-0.02[-0.09,0.05]$ & \\
\hline Landoni 2007 & -0.1 & 0.15 & 59 & -0.1 & 0.16 & 61 & $27.9 \%$ & $0.00[-0.06,0.06]$ & \\
\hline Lorsomradee 2006 & -0.2 & 0.19 & 160 & -0.1 & 0.13 & 160 & $32.5 \%$ & $-0.10[-0.14,-0.06]$ & 늘 \\
\hline Total $(95 \% \mathrm{Cl})$ & & & 306 & & & 306 & $100.0 \%$ & $-0.04[-0.10,0.02]$ & \\
\hline \multicolumn{7}{|c|}{$\begin{array}{l}\text { Heterogeneity: } \mathrm{Tau}^{2}=0.00 ; \mathrm{Chi}^{2}=11.11, \mathrm{df}=3(P=0.01) ; \mathrm{I}^{2}=73 \% \\
\text { Test for overall effect: } Z=1.33(P=0.18)\end{array}$} & & & $\begin{array}{ccccc}-0.5 & -0.25 & 0 & 0.25 & 0.5 \\
\text { Favours Volatile } & \text { Favours TIVA }\end{array}$ \\
\hline
\end{tabular}

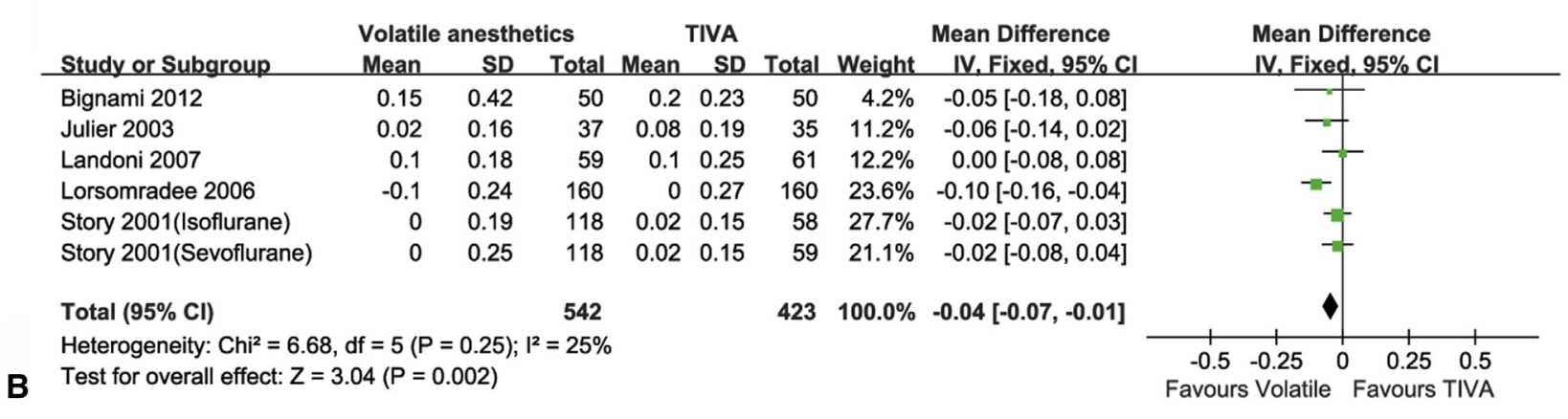

\begin{tabular}{|c|c|c|c|c|c|c|c|c|c|}
\hline \multirow[b]{2}{*}{ Study or Subgroup } & \multicolumn{3}{|c|}{ Volatile anesthetics } & \multicolumn{2}{|c|}{ TIVA } & \multirow[b]{2}{*}{ Total } & \multirow[b]{2}{*}{ Weight } & \multirow{2}{*}{$\begin{array}{l}\text { Mean Difference } \\
\text { IV, Random, } 95 \% \mathrm{Cl}\end{array}$} & \multirow{2}{*}{$\begin{array}{l}\text { Mean Difference } \\
\text { IV. Random, } 95 \% \mathrm{Cl}\end{array}$} \\
\hline & Mean & SD & Total & Mean & SD & & & & \\
\hline Bignami 2012 & 0.3 & 0.49 & 50 & 0.28 & 0.39 & 50 & $9.7 \%$ & $0.02[-0.15,0.19]$ & \\
\hline Julier 2003 & 0.03 & 0.17 & 37 & 0.06 & 0.19 & 35 & $18.2 \%$ & $-0.03[-0.11,0.05]$ & \\
\hline Landoni 2007 & 0.1 & 0.24 & 59 & 0.2 & 0.47 & 61 & $13.0 \%$ & $-0.10[-0.23,0.03]$ & \\
\hline Lorsomradee 2006 & -0.1 & 0.24 & 160 & 0.1 & 0.36 & 160 & $20.1 \%$ & $-0.20[-0.27,-0.13]$ & - \\
\hline Story 2001(Isoflurane) & 0 & 0.17 & 118 & 0.04 & 0.22 & 59 & $20.4 \%$ & $-0.04[-0.10,0.02]$ & \\
\hline Story 2001(Sevoflurane) & 0.01 & 0.32 & 118 & 0.04 & 0.22 & 59 & $18.6 \%$ & $-0.03[-0.11,0.05]$ & \\
\hline Total $(95 \% \mathrm{Cl})$ & & & 542 & & & 424 & $100.0 \%$ & $-0.07[-0.14,-0.00]$ & \\
\hline $\begin{array}{l}\text { Heterogeneity: } \operatorname{Tau}^{2}=0.0 \\
\text { Test for overall effect: } Z=\end{array}$ & $\begin{array}{l}\text { Chi }{ }^{2}=1 \\
99(P=\end{array}$ & $\begin{array}{l}03, \mathrm{df}= \\
05)\end{array}$ & $(P=0$ & US), & -1 & & & & 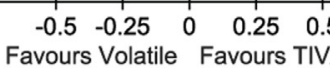 \\
\hline
\end{tabular}

FIGURE 4. Forest plot of weighted mean differences in the change of serum creatinine level from baseline at intensive care unit arrival (A), on the first postoperative day (B), and on the second postoperative day (C). TIVA, Total intravenous anesthesia; $I V$, instrumental variable; $C I$, confidence interval; $S D$, standard deviation.

postoperative day. With regard to the change from baseline, patients who received volatile anesthetics had a significantly smaller rise in creatinine level on the first and second postoperative days. It has been reported that the degree of creatinine elevation is more important than the absolute level of creatinine for estimating death risk in patients with AKI, and even a small increase in creatinine after cardiac surgery is correlated with increased duration and cost of hospitalization and with the short-term risk of mortality. ${ }^{33-35}$ In addition, renal injury was also assessed with the postoperative measurement of serum cystatin $\mathrm{C}$ concentration. As opposed to serum creatinine concentration, cystatin $\mathrm{C}$ level is independent of age, sex, muscle mass, or dietary factors, and it more readily and accurately reflects even mild renal dysfunction. ${ }^{36}$ Results from our current systematic review suggested that there was a borderline significantly lower level of cystatin $\mathrm{C}$ in the volatile anesthetic group. Consistent with improvement in postoperative renal function, volatile anesthetic administration significantly reduced the incidences of prolonged
ICU stay and prolonged hospitalization and slightly, although not significantly, decreased mortality.

Except for a single study with isoflurane, the volatile anesthetic choice for patients in the studies included in this review was desflurane or sevoflurane. To address the question of whether the choice of anesthetic regimen might influence patients' outcome, we conducted a subgroup analysis. We found a significant trend toward reduced AKI among the patients who received sevoflurane, whereas no such trend was evident among the patients who received desflurane. In animal studies, Lee and coworkers ${ }^{37}$ has shown that the degree of renal protection by desflurane was significantly less than that conferred by sevoflurane, which was thought be related to their different properties in promoting attenuation of cellular injury and functional recovery. ${ }^{38}$ Because of the limited number of trials with desflurane, however, we failed to determine whether desflurane and sevoflurane were associated with different changes in renal biomarkers.

The strengths of this systematic review include the use of strict methodologic inclusion criteria, a quality assessment 


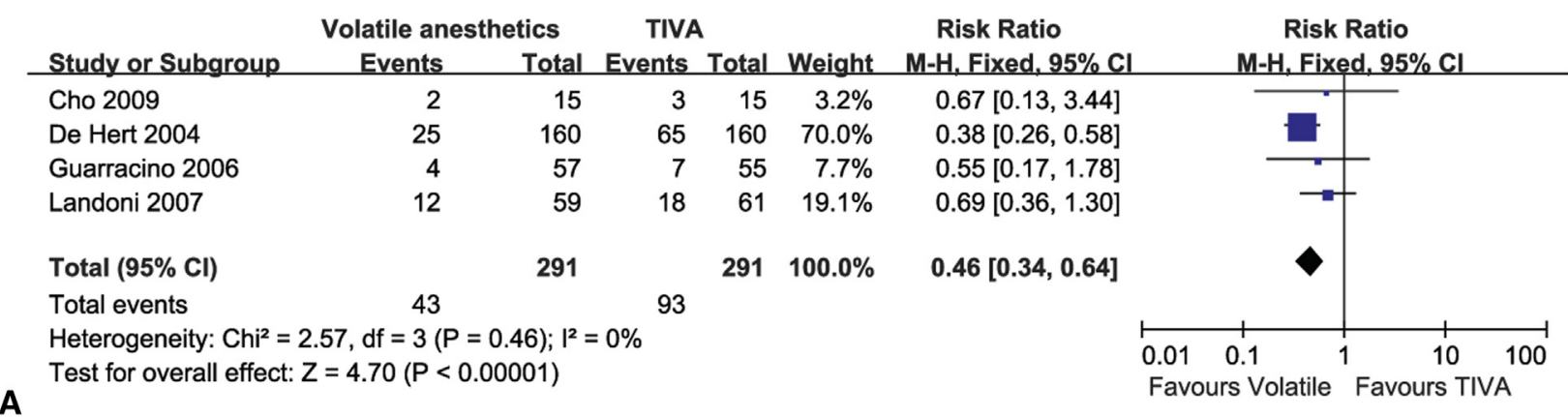

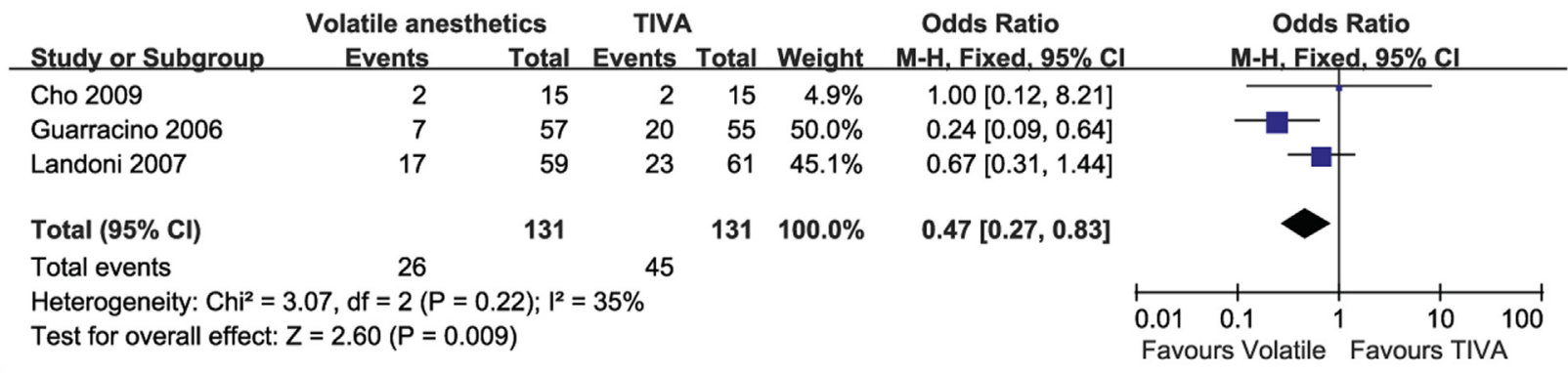

B

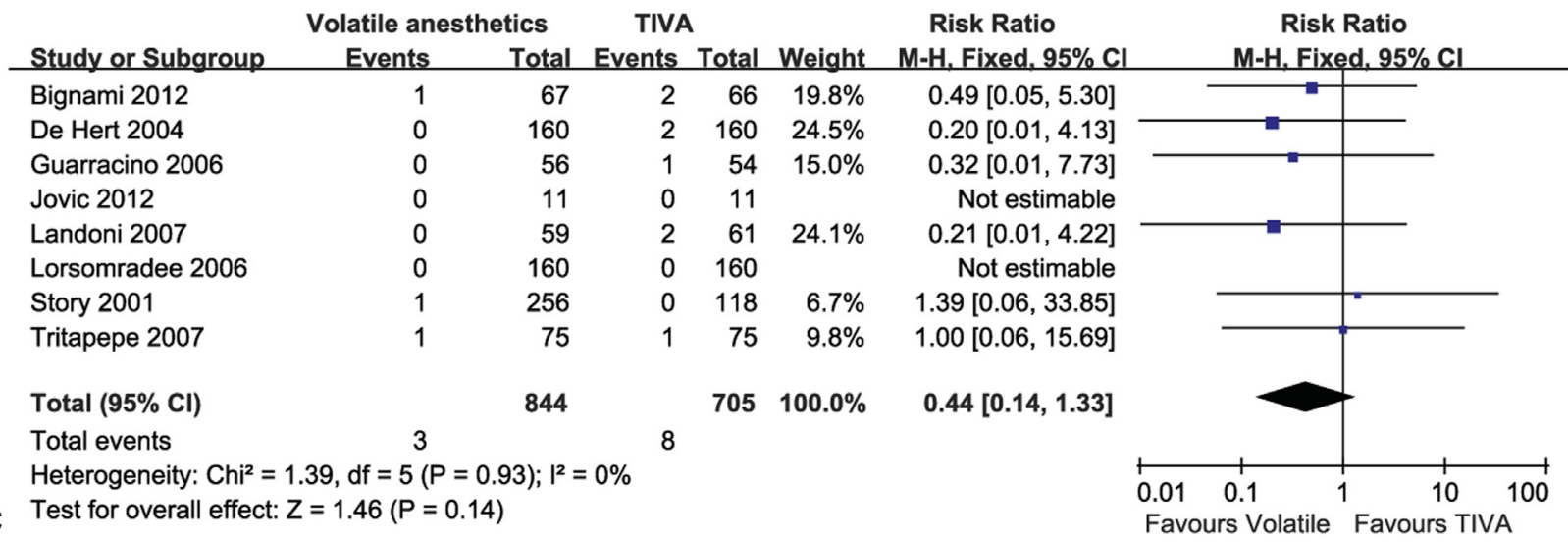

FIGURE 5. Forest plot of relative risks in the incidences of prolonged intensive care unit stay (A), prolonged hospitalization (B), and all-cause mortality (C). TIVA, Total intravenous anesthesia; $M-H$, Mantel-Haenszel (log-rank); $C I$, confidence interval.

of all clinical trials, and its ability to garner a sufficiently large study population in this area dominated by relatively small studies. On the other hand, there are several potential limitations of this meta-analysis. First, as mentioned previously, various definitions have been proposed for postoperative AKI. Although there was not considerable heterogeneity among the included studies, these data do not yet allow any firm conclusions. Second, there was a significant level of variability in the time and duration of volatile anesthetic administration, and because of a lack of available data in a usable format, a subgroup analysis could not be performed. Third, publication bias cannot be excluded. We did not test for publication bias with funnel plots or other statistical tests, because such tests are unreliable in the presence of a small number of studies. We did, however, undertake an effort to minimize publication bias by including broad search and inclusion criteria, hand searching the references, and contacting the authors of the primary trials.

We thank the investigators of the original studies for generously sharing their data with us.

\section{References}

1. Rosner MH, Okusa MD. Acute kidney injury associated with cardiac surgery. Clin J Am Soc Nephrol. 2006;1:19-32.

2. Shaw A. Update on acute kidney injury after cardiac surgery. J Thorac Cardiovasc Surg. 2012;143:676-81.

3. Koyner JL, Bennett MR, Worcester EM, Ma Q, Raman J, Jeevanandam V, et al Urinary cystatin $\mathrm{C}$ as an early biomarker of acute kidney injury following adult cardiothoracic surgery. Kidney Int. 2008;74:1059-69.

4. Fang Y, Ding X, Zhong Y, Zou J, Teng J, Tang Y, et al. Acute kidney injury in a Chinese hospitalized population. Blood Purif. 2010;30:120-6.

5. Mitter N, Shah A, Yuh D, Dodd-O J, Thompson RE, Cameron D, et al. Renal injury is associated with operative mortality after cardiac surgery for women and men. J Thorac Cardiovasc Surg. 2010;140:1367-73.

6. Bonventre JV, Zuk A. Ischemic acute renal failure: an inflammatory disease? Kidney Int. 2004;66:480-5. 
7. Bellomo R, Auriemma S, Fabbri A, D’Onofrio A, Katz N, McCullough PA, et al. The pathophysiology of cardiac surgery-associated acute kidney injury (CSA-AKI). Int J Artif Organs. 2008;31:166-78.

8. Landoni G, Biondi-Zoccai GG, Zangrillo A, Bignami E, D’Avolio S, Marchetti C, et al. Desflurane and sevoflurane in cardiac surgery: a metaanalysis of randomized clinical trials. J Cardiothorac Vasc Anesth. 2007;21: 502-11.

9. Minguet G, Joris J, Lamy M. Preconditioning and protection against ischaemia-reperfusion in non-cardiac organs: a place for volatile anaesthetics? Eur J Anaesthesiol. 2007;24:733-45.

10. Landoni G, Bignami E, Oliviero F, Zangrillo A. Halogenated anaesthetics and cardiac protection in cardiac and non-cardiac anaesthesia. Ann Card Anaesth. 2009; 12:4-9.

11. Zaugg M, Lucchinetti E, Uecker M, Pasch T, Schaub MC. Anaesthetics and cardiac preconditioning. Part I. Signalling and cytoprotective mechanisms. Br J Anaesth. 2003;91:551-65.

12. Landoni G, Fochi O, Torri G. Cardiac protection by volatile anaesthetics: a review. Curr Vasc Pharmacol. 2008;6:108-11.

13. Kim M, Park SW, Kim M, D’Agati VD, Lee HT. Isoflurane activates intestinal sphingosine kinase to protect against renal ischemia-reperfusion-induced liver and intestine injury. Anesthesiology. 2011;114:363-73.

14. Lee HT, Chen SW, Doetschman TC, Deng C, D'Agati VD, Kim M. Sevoflurane protects against renal ischemia and reperfusion injury in mice via the transforming growth factor- $\beta_{1}$ pathway. Am J Physiol Renal Physiol. 2008; 295:F128-36.

15. Jia P, Teng J, Zou J, Fang Y, Zhang X, Bosnjak ZJ, et al. miR-21 contributes to xenon-conferred amelioration of renal ischemia-reperfusion injury in mice. Anesthesiology. 2013;119:621-30.

16. Julier K, da Silva R, Garcia C, Bestmann L, Frascarolo P, Zollinger A, et al. Preconditioning by sevoflurane decreases biochemical markers for myocardial and renal dysfunction in coronary artery bypass graft surgery: a double-blinded, placebo-controlled, multicenter study. Anesthesiology. 2003; 98:1315-27.

17. Sindhvananda W, Phisaiphun K, Prapongsena P. No renal protection from volatileanesthetic preconditioning in open heart surgery. J Anesth. 2013;27:48-55.

18. Jüni P, Altman DG, Egger M. Systematic reviews in health care: assessing the quality of controlled clinical trials. BMJ. 2001;323:42-6.

19. Hozo SP, Djulbegovic B, Hozo I. Estimating the mean and variance from the median, range, and the size of a sample. BMC Med Res Methodol. 2005;5:13.

20. Bignami E, Landoni G, Gerli C, Testa V, Mizzi A, Fano G, et al. Sevoflurane vs. propofol in patients with coronary disease undergoing mitral surgery: a randomised study. Acta Anaesthesiol Scand. 2012;56:482-90.

21. Cho EJ, Yoon JH, Hong SJ, Lee SH, Sim SB. The effects of sevoflurane on systemic and pulmonary inflammatory responses after cardiopulmonary bypass. J Cardiothorac Vasc Anesth. 2009;23:639-45.

22. De Hert SG, Van der Linden PJ, Cromheecke S, Meeus R, ten Broecke PW, De Blier IG, et al. Choice of primary anesthetic regimen can influence intensive care unit length of stay after coronary surgery with cardiopulmonary bypass. Anesthesiology. 2004;101:9-20.
23. Guarracino F, Landoni G, Tritapepe L, Pompei F, Leoni A, Aletti G, et al. Myocardial damage prevented by volatile anesthetics: a multicenter randomized controlled study. J Cardiothorac Vasc Anesth. 2006;20:477-83.

24. Jovic M, Stancic A, Nenadic D, Cekic O, Nezic D, Milojevic P, et al. Mitochondrial molecular basis of sevoflurane and propofol cardioprotection in patients undergoing aortic valve replacement with cardiopulmonary bypass. Cell Physiol Biochem. 2012;29:131-42.

25. Landoni G, Calabrò MG, Marchetti C, Bignami E, Scandroglio AM, Dedola E, et al. Desflurane versus propofol in patients undergoing mitral valve surgery. J Cardiothorac Vasc Anesth. 2007;21:672-7.

26. Lorsomradee S, Cromheecke S, Lorsomradee S, De Hert SG. Effects of sevoflurane on biomechanical markers of hepatic and renal dysfunction after coronary artery surgery. J Cardiothorac Vasc Anesth. 2006;20:684-90.

27. Story DA, Poustie S, Liu G, McNicol PL. Changes in plasma creatinine concentration after cardiac anesthesia with isoflurane, propofol, or sevoflurane: a randomized clinical trial. Anesthesiology. 2001;95:842-8.

28. Tritapepe L, Landoni G, Guarracino F, Pompei F, Crivellari M, Maselli D, et al. Cardiac protection by volatile anaesthetics: a multicentre randomized controlled study in patients undergoing coronary artery bypass grafting with cardiopulmonary bypass. Eur J Anaesthesiol. 2007;24:323-31.

29. Swyers T, Redford D, Larson DF. Volatile anesthetic-induced preconditioning. Perfusion. 2014;29:10-5.

30. Gravel NR, Searle NR, Taillefer J, Carrier M, Roy M, Gagnon L. Comparison of the hemodynamic effects of sevoflurane anesthesia induction and maintenance vs TIVA in CABG surgery. Can J Anaesth. 1999;46:240-6.

31. Borthwick E, Ferguson A. Perioperative acute kidney injury: risk factors, recognition, management, and outcomes. BMJ. 2010;341:c3365.

32. Schetz M, Bove T, Morelli A, Mankad S, Ronco C, Kellum JA. Prevention of cardiac surgery-associated acute kidney injury. Int J Artif Organs. 2008;31:179-89.

33. Cuhaci B. More data on epidemiology and outcome of acute kidney injury with AKIN criteria: benefits of standardized definitions, AKIN and RIFLE classifications. Crit Care Med. 2009;37:2659-61.

34. Brown JR, Cochran RP, Dacey LJ, Ross CS, Kunzelman KS, Dunton RF, et al. Perioperative increases in serum creatinine are predictive of increased 90-day mortality after coronary artery bypass graft surgery. Circulation. 2006; 114(1 Suppl):I409-13.

35. Falvo A, Horst HM, Rubinfeld I, Blyden D, Brandt MM, Jordan J, et al. Acute renal failure in cardiothoracic surgery patients: what is the best definition of this common and potent predictor of increased morbidity and mortality. Am J Surg. 2008;196:379-83.

36. Laterza OF, Price CP, Scott MG. Cystatin C: an improved estimator of glomerular filtration rate? Clin Chem. 2002;48:699-707.

37. Lee HT, Ota-Setlik A, Fu Y, Nasr SH, Emala CW. Differential protective effects of volatile anesthetics against renal ischemia-reperfusion injury in vivo. Anesthesiology. 2004;101:1313-24.

38. Preckel B, Schlack W, Comfère T, Obal D, Barthel H, Thamer V. Effects of enflurane, isoflurane, sevoflurane and desflurane on reperfusion injury after regional myocardial ischaemia in the rabbit heart in vivo. Br J Anaesth. 1998; 81:905-12. 